RESEARCH ARTICLE

\title{
A Hepatitis C Sentinel Seroprevalence, Cape Coast, Ghana
}

\author{
Ato Kwamena Tetteh ${ }^{1}$, Godsway Aglagoh ${ }^{1}$, Charles Baffe ${ }^{1}$, Sadick Arthur ${ }^{1}$, Prince Bram ${ }^{1}$, Gifty Rhodalyn \\ Tetteh $^{2}$, Edward Agyarko ${ }^{3}$ \\ ${ }^{1}$ Metropolitan Hospital, Laboratory Department, Cape Coast, CR, Ghana \\ ${ }^{2}$ Department of Forensic Sciences, University of Cape Coast, Cape Coast, CR, Ghana \\ ${ }^{3}$ Anglican University College of Technology, Department of Community Medicine and Health, Nkoranza, BA, Ghana
}

\begin{abstract}
Objectives: Sentinel studies have targeted prenatal women as they are largely descriptive of the reproductive bracket, and prevalence data may be extended to the general population. This study determined the seroprevalence of hepatitis $\mathrm{C}$ virus ( $\mathrm{HCV}$ ) infection among pregnant women attending antenatal clinic at the Cape Coast Metropolitan Hospital.
\end{abstract}

Methods: Whole blood samples were collected from 258 pregnant women aged between 15-45 years, from September 1st through December 31st, 2018. Separated plasma was stored and later screened for HCV antibodies.

Results: Out of the total, $2.7 \%$ (7/258) tested seropositive for HCV antibodies. The 20 - 29 years age group recorded the highest prevalence of $1.6 \%\left(4 / 258, \chi^{2}=4.260, p=0.513\right)$. According to the $2018 \mathrm{HIV}$ Sentinel Surveillance report, the prevalence of HIV infection in the Cape Coast Metropolitan Area was 3.4\% (17/500). Also, the prevalence of HIV among the $20-29$ years age group was $1.8 \%$ (9/500). The $15-24$ years, proxy group for new infections recorded $0.8 \%$ (2/258) for HCV and $0.2 \%$ (1/500) for HIV infection.

Conclusions: The seroprevalence determined in this study is classified 'intermediate,' according to the WHO guidelines for the care and treatment of persons diagnosed with chronic hepatitis $C$ virus infection.

The WHO currently does not recommend routine HCV testing for pregnant women; however, this study highlights the need for urgent public health interventions aimed at reducing the infection rate. J Microbiol Infect Dis 2020; 10(1):5255.

Keywords: Hepatitis C, Sentinel, Pregnant women, Antenatal screening, Seroprevalence

\section{INTRODUCTION}

Hepatitis $\mathrm{C}$ virus ( $\mathrm{HCV}$ ) infection is one of the common chronic blood-borne diseases, similar to hepatitis B and HIV infection globally [1]. Following the identification of the hepatitis $C$ virus in 1989 [2], and the development of specific diagnostic assays, hepatitis $C$ infection is now recognized as a worldwide public health problem which affect $1.3-8.0 \%$ of the world's population. Globally, the prevalence of hepatitis $\mathrm{C}$ virus (HCV) infection was estimated to be between 1.2 and $3.8 \%$ according to World Health Organization (WHO) Global Burden of
Disease, with more than 185 million people infected worldwide [3].

Studies conducted in specific sub-Saharan African countries estimated a high prevalence of HCV (6.0\%) in central African countries. In North Africa, Egypt has the highest $\mathrm{HCV}$ prevalence of $17.5 \%$, followed by Morocco with $7.7 \%$. In spite of the advances in the pathogenesis, treatment, and public health response to hepatitis $C$ virus $(\mathrm{HCV})$, pregnancy related $\mathrm{HCV}$ has been deserted. Women infected with HCV have a 2$8 \%$ risk of viral transmission to their infant but the mechanism and timing of mother to child transmission (MTCT) are not well understood, 
nor is the natural history of the illness in pregnant women and their offspring [4]. A study conducted among pregnant women in Nigeria, found the prevalence of HCV to be between $1.4 \%$ for women aged between 20 and 35 years old and $11.1 \%$ for women aged less than 20 years old [3]. In Ghana, there exist no clear data for HCV prevalence among pregnant women, however, a recent systematic review conducted by Agyemang et al. [5], mainly among low risk individuals found a prevalence rate of $3.0 \%$. Among pregnant women in Ghana, anti-HCV seroprevalence was estimated as $4.6 \%$ in a systematic review and meta-analysis [5]. This study sought to determine seroprevalence of hepatitis $\mathrm{C}$ and trends in the Cape Coast Metropolitan Hospital, which is an existing HIV Sentinel Site.

\section{METHODS}

\section{Study Setting}

The Cape Coast Metropolitan Hospital (CCMH) is a large public hospital, and serves as the first point of care for the entire Cape Coast Metropolis. Its bed capacity could exceed 250 but it is usually 180 , and about $60-70 \%$ filled. It receives referred patients from polyclinics/health centers, and also from the Cape Coast Teaching Hospital when the facility is overburdened. Cape Coast is the capital of the Central Region of Ghana, with a population of 169,894 . It is located on longitude $1^{\circ} 15^{\prime} \mathrm{W}$ and latitude $5^{\circ} 06^{\prime} \mathrm{N}$. It occupies an Area of approximately $122 \mathrm{~km} 2$. Currently the $\mathrm{CCMH}$ serves as the Sentinel Site for the nationwide HIV surveillance for Cape Coast, and Tuberculosis and Cholera referral center for the Central Region of Ghana.

\section{Study Design}

The study was cross sectional and was run with randomly (Random integer generator, Random.org) selected 258 stored plasma samples used for the 2018 nationwide HIV Sentinel Surveillance (HSS).

The study sampled pregnant women, aged 1545 years, who attended antenatal clinic for the first time during the survey period (September December, 2018). Pregnant women who met these criteria had their blood samples collected until the end of the survey period. Those reporting for repeated visit during the survey period were not sampled for the second time.
The one-step immunochromatographic test kit (Advanced Quality ${ }^{\mathrm{TM}}$, InTech Products Inc., Sensitivity $=100 \%$, Specificity $=97-99 \%$ ) for the HCV serologic test was used.

Manufacturer's assay procedure was adhered to as follows: Ten $\mu \mathrm{L}$ plasma volume was dispensed to the upper edge of the sample pad of the test strip by using a plastic dropper provided in the pouch. Following this, two drops of the diluent added to the lower edge. Results were interpreted after 15 minutes.

\section{Ethical Consideration}

Permission was granted from the Hospital to carry out this study for informational/educational purposes and to provide preliminary data on the seroprevalence of HCV. Participants' identity was concealed as much as possible during the analysis. Therefore, new path numbers were assigned to samples selected for HCV testing and analysis. This study was not expected to cause any harm to participants in accordance with the Declaration of Helsinki (1964). Participants who were seropositive were given appropriate care through their routine antenatal clinic.

\section{Data Analysis}

Age and test outcome (sero-positive/negative) for all pregnant women screened, were initially recorded and entered into the Statistical Package for Social Sciences Software (SPSS, Version 23.0, IBM, https://www.ibm.com) for analysis. Data was analyzed according to age groups and serologic test outcome for HCV. Chi square $(x 2)$ and $p<0.05$ considered to be significant at $95 \%$ confidence were estimated.

\section{RESULTS}

A total of 258 pregnant women aged between 15 and 44 (mean age $=26.79$, standard error $=$ 0.386, 95\%Cl: 26.08-27.52) were included in this study. Out of the total, $31.4 \%(81 / 258)$ were in the 25-29 years age group. The 40-44 years age group constituted only $2.3 \%(6 / 25)$ of the total. The 15-24-year group classified as the proxy group for new infections constituted $36.8 \%$ (95/258) (Figure 1).

In all, $2.7 \%(7 / 258)$ were seropositive with the remaining being seronegative (Table 1). Seropositivity was found mainly among the 2024- and 25-29-year groups $\left(x^{2}=4.260, p=\right.$ 
0.513). The proxy group (15-24 years) for new infections recorded $0.8 \%(2 / 258)$. The HIV status for these groups were not determined for this study, however, the 2018 HIV prevalence (from the 2018 sentinel surveillance report) for the entire Cape Coast Metropolitan Area was estimated to be $3.7 \%(17 / 500)$, which is a $0.4 \%$ increase from the previous year.

Table 1. Sero-prevalence of HCV among the participants

\begin{tabular}{cccc}
\hline \multicolumn{4}{c}{ HCV Tests Results } \\
\hline Age (Year) & Positive (\%) & Negative & Total \\
$15-19$ & $0(0.0)$ & $36(14.0)$ & 36 \\
$20-24$ & $2(0.8)$ & $57(22.1)$ & 59 \\
$25-29$ & $4(1.6)$ & $77(29.8)$ & 81 \\
$30-34$ & $0(0.0)$ & $49(19.0)$ & 49 \\
$35-39$ & $1(0.4)$ & $26(10.1)$ & 27 \\
$40-44$ & $0(0.0)$ & $6(2.3)$ & 6 \\
Total & $7(2.7)$ & $251(97.3)$ & 258 \\
\hline
\end{tabular}

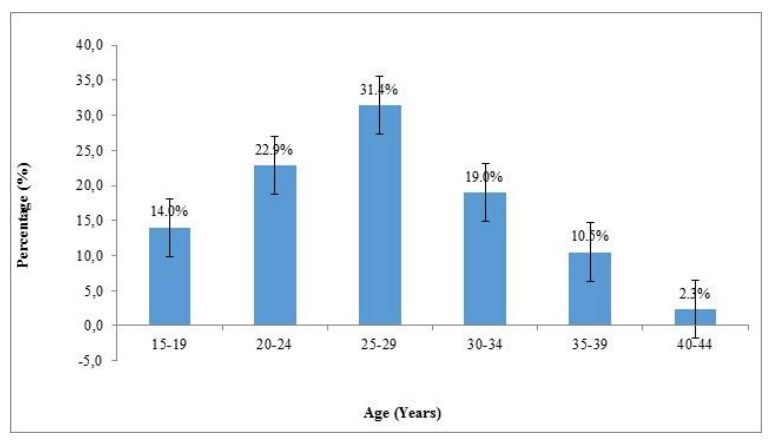

Figure 1: Age distribution among pregnant women

\section{DISCUSSION}

In this study, plasma samples from pregnant women who attended antenatal care clinic during the survey period were screened for hepatitis $\mathrm{C}$ antibodies. Currently the WHO does not recommend routine testing for Hepatitis $C$ (HCV) among pregnant women [6]. Therefore, in Ghana, HCV testing is not included in the initial routine tests requested for all pregnant women in government funded antenatal centers nationwide. Often clinically silent initially, chronic $\mathrm{HCV}$ infection predisposes to eventual development of liver fibrosis and hepatocellular carcinoma, as well as various extra-hepatic impediments [7-9]. Regardless of the advances in the pathogenesis, treatment, and public health response to $(\mathrm{HCV})$, pregnancy related $\mathrm{HCV}$ has been neglected. Meanwhile, a markedly improved public health response to this population is needed. Of 258 pregnant women screened for HCV, $2.7 \%$ was seropositive. In a similar much larger study in Rwanda, which screened 12,903 pregnant women from 30 HIV sentinel sites, a prevalence of $2.6 \%$ was estimated [3].

The age group with the highest HCV prevalence was the 25-29 years. This overall prevalence among pregnant women in our setting was relatively higher compared to retrospective findings published by Kopilovic et al. [4] in some European countries. On the other hand, our findings for HCV prevalence among pregnant women at our setting is higher than findings reported from Nigeria (1.5\%), Malawi $(2.0 \%)$, United Kingdom (0.8\%), Brazil (0.2\%) and Central African region (2.0\%), however, lower than overall estimated prevalence for subSaharan African countries (3.0\%) [3]. The explanation for these variations could be due to differences in geographical patterns and varying physician test ordering practices, care-seeking and disclosure behaviors of pregnant women, or factors that are related to HCV risk [10].

Hepatitis C is known to have a $10 \%$ chance of vertical transmission [11,12], compared with that of HIV, which varies between 15 and $45 \%$ in the absence of intervention in-utero, during labor or breastfeeding [13]. Existing standard treatment are known to have contraindications during pregnancy making it difficult for physicians to administer them [14,15].

In Ghana, HCV testing is usually performed for blood donors to prevent haemotransfusion transmission. Generally, HCV testing in Ghana has challenges because it is not standardized, tied with the fact that the diagnostic market is flooded with many brands from different production companies. These, coupled with scanty information about the public health relevance does not support advocacy to include its screening among pregnant women at antenatal clinics, and among the general population. Supplementary testing is currently unavailable routinely in Ghana and was 
therefore not performed for this study. In this regard, the study could not eliminate falsepositive antibody results.

\section{Conclusion}

This study has documented for the first-time seropositive HCV infection among antenatal care attendants in the Cape Coast Metropolitan Hospital. Hepatitis C virus infection could be public health challenge among pregnant women in Ghana if the situation is not addressed. The results highlight the need for urgent public health interventions aimed at reducing the infection rate. This study provides an avenue to inform policymakers and stakeholders, the need to accelerate progress in treatment access and global prevention of HCV infection. These must include efforts to increase awareness and knowledge about HCV transmission dynamics, targeting the screening of high-risk groups and providing treatment for affected individuals. Advanced studies of HCV on this group and in the general population are needed to explore detection of antigens and antibodies and how they inform the diagnosis of acute and active $\mathrm{HCV}$ infections. Further research is also needed to understand fully the population factors underlying this high $\mathrm{HCV}$ incidence.

\section{ACKNOWLEDGMENTS}

We are indebted to laboratory staff and National Service Personnel at the Cape Coast Metropolitan Hospital who helped to collect and process samples for serological testing.

Declaration of Conflicting Interests: The authors declare that they have no conflict of interest.

Financial Disclosure: No financial support was received.

\section{REFERENCES}

1. Lavanchy D. Evolving epidemiology of hepatitis C virus. Clin Microbiol Infect 2011; 17(2): 107-115.

2. Choo QL, Kuo G, Weiner AJ, et al. Isolation of a cDNA clone derived from a blood-borne non-A, non-B viral hepatitis genome. Science 1989; 244 (4902): 359-362.

3. Mutagoma $M$, Balisanga $H$, Sebuhoro $D$, et al. Hepatitis C virus and HIV co-infection among pregnant women in Rwanda. BMC infectious diseases 2017; 17(1): 167.

4. Kopilović B, Poljak M, Seme K, Klavs I. Hepatitis $C$ virus infection among pregnant women in
Slovenia: study on 31,849 samples obtained in four screening rounds during 1999, 2003, 2009 and 2013. Eurosurveillance 2015; 20(22): 21144.

5. Agyeman AA, Ofori-Asenso $R$, Mprah A, Ashiagbor $G$. Epidemiology of hepatitis $C$ virus in Ghana: a systematic review and meta-analysis. BMC infectious diseases 2016; 16(1): 391.

6. World Health Organization. Guidelines for the care and treatment of persons diagnosed with chronic hepatitis $C$ virus infection. Geneva: World Health Organization 2018; Licence: CC BY-NC-SA 3.0 IGO.

7. Di Bisceglie AM, Order SE, Klein JL, et al. The role of chronic viral hepatitis in hepatocellular carcinoma in the United States. Am Journal of Gastroenterology 1991; 86(3): 335-338.

8. The Global burden of Hepatitis C working Group Global burden of disease (GBD) for hepatitis C. J Clin Pharmacol 2004; 44: 20-29.

9. Singal AG, Lampertico $P$, Nahon $P$. Epidemiology and surveillance for hepatocellular carcinoma: New trends. J Hepatol 2020; 72(2):250-261.

10. Blasig A, Wagner EC, Pi D, et al. Hepatitis C infection among pregnant women in British Columbia: reported prevalence and critical appraisal of current prenatal screening methods. Canadian J Public Health 2011; 102(2): 98-102.

11. Thomas SL, Newell ML, Peckham CS, Ades AE, Hall AJ. A review of hepatitis $C$ virus ( $\mathrm{HCV}$ ) vertical transmission: risks of transmission to infants born to mothers with and without HCV viraemia or human immunodeficiency virus infection. Int J Epidemiol 1998; 27(1):108-117.

12. Campion A, Larouche A, Fauteux-Daniel S, Soudeyns $H$. Pathogenesis of hepatitis $C$ during pregnancy and childhood. Viruses 2012; 4:35313550.

13. Barral MF, Oliveira GR, Lobato RC, MendozaSassi RA, Martínez AM, Gonçalves CV. Risk factors of HIV-1 vertical transmission (VT) and the influence of antiretroviral therapy (ART) in pregnancy outcome. Revista do Instituto de Medicina Tropical de São Paulo 2014; 56(2):133138.

14. Labarga P, Pinilla J, Cachorro I, del Prado YR. Infant of 22 months of age with no anomalies born from a HCV-and HIV-infected mother under treatment with pegylated interferon, ribavirin and antiretroviral therapy during the first 16 weeks of pregnancy. Reproductive Toxicology 2007; 1; 24(3-4):414-416.

15. Toussaint-Miller KA, Andres J. Treatment considerations for unique patient populations with HCV genotype 1 infection. Annals of Pharmacotherapy. 2015; 49(9):1015-1030. 